\title{
LA COLABORACIÓN ENTRE MAESTROS/MAESTRAS Y PADRES/MADRES PARA ATENDER A LA DIVERSIDAD EN LAS INSTITUCIONES EDUCATIVAS
}

\author{
Ronald Soto Calderón \\ Universidad de Costa Rica \\ Francisco Javier Hinojo Lucena \\ Universidad de Granada (España)
}

\author{
Recibido 13-II-2004 • Aceptado 9-III-2004
}

\begin{abstract}
Resumen: En este artículo se resalta la importancia de un trabajo conjunto entre los padres y madres de familia de personas con necesidades educativas especiales y los docentes, y las diferentes formas en que esta labor se puede realizar.

También se comentan los diferentes planos en los que se puede llevar a cabo un trabajo cooperativo, de tal manera que lo importante siempre se enfoque a la atención educativa de los estudiantes con NEE, se mencionan también algunos puntos que pueden ser tomados en cuenta para evitar las diferencias en las actuaciones tanto de los padres y madres de familia como de los docentes, de los estudiantes con NEE en las situaciones que le competen a las instituciones.

Se considera por parte de los autores, que un trabajo coordinado, colaborativo y consciente de todas las partes que participan en el desarrollo del proceso aprendizaje de personas con NEE, siempre se verá reflejado en el éxito y realización de los potencialidades de todas las personas, y el derecho a la participación de las personas en igualdad y equiparación de oportunidades.
\end{abstract}

Palabras clave: Necesidades Educativas Especiales, Trabajo Padres Maestros, Personas con Necesidades Educativas Especiales.

\section{Introducción}

El alumnado con necesidades educativas especiales es aquel que presenta algún problema de aprendizaje a lo largo de su escolarización y que demanda una atención más específica, en cuanto a que necesita un abordaje educativo especial para el desarrollo de sus capacidades, así como mayores recursos educativos de los necesarios para los compañeros de su edad, lo que le permita su incorporación social.

¿Qué sería de nosotros sin ayuda profesional? Seguramente esta pregunta se la hacen muchas familias cuando se encuentran ante un hijo con necesidades educativas especiales y pensaron: ¿qué vamos a hacer ahora o a quién nos podemos dirigir?

Son muchas las dificultades que deben pasar tanto los padres como los niños con discapacidades, cuando se encuentran ante un mundo que más que ayudarles a integrar a sus hijos, no hace más que poner impedimentos para su desarrollo como cualquier otro niño sin ninguna discapacidad. Pero 


\begin{abstract}
This article highlights both the importance of a the importance of a joint work between the parents and teachers of people with special educational needs, and the different forms this work can be done. Also, the article discusses the different educational attentions of the student areas in which a cooperative work can be carried out, in such a way that what is important is the approach to the educational attention of the students with special educational needs in those situations which are the sole competence of the institutions. This paper also mentions some points that can be taken out to avoid the differences in the performances of the parents and teachers of the students with Special Educational Needs in those situations which are the sole competence of the institutions. The authors consider that which a coordinated, collaborative and conscious work of all the people and their right to participate in the development of the process of learning of people with Special Educational Needs, will always be reflected in the success and accomplishment of the potentialities of all the people, and there right to the participate in equality and comparison of opportunities.
\end{abstract}

Key words: Special Educational Needs, Parents Teachers Work, People With Special Educational Needs. afortunadamente existen organismos y legislaciones que apoyan a estas familias y la plena integración de sus hijos.

Se pretende cerrar el tema específicamente en la relación que los padres y madres de alumnos/alumnas establecen con la escuela y esta con la familia una vez que ha llegado el momento de la escolarización de sus hijos. Qué tipos de dificultades se van a encontrar, qué derechos y obligaciones como padres de alumnos con necesidades educativas especiales tienen en el ámbito de la educación y qué pueden hacer los profesionales de la enseñanza, la ley y ellos mismos por este tipo de niños.

Se expondrán qué variables influyen en las actuaciones de los padres en colaboración con la escuela y con sus hijos, y qué programas existen para que los padres puedan llevar a cabo en el hogar y ayudar a sus hijos para que consigan un mejor desarrollo de todas sus capacidades.

\section{Consideraciones previas: los padres y madres de alumnos/ alumnas con N.E.E. y la confirmación de tal diagnóstico}

Para que pueda existir una plena colaboración entre los maestros y los padres de los alumnos con NEE (necesidades educativas especiales), es preciso que los maestros conozcan el proceso que generalmente siguen los padres hasta confirmar el diagnóstico de su hijo y llegar a aceptarlo.

Cuando nace un niño considerado de alto riesgo, empieza para los padres, un proceso entre la angustia y la esperanza, que les llevará de un médico a otro en busca de un diagnóstico que no confirme la sospecha que tienen o que niegue la deficiencia.

El médico establece el diagnóstico o juicio diagnóstico, donde se manifiesta la deficiencia del niño.

Una nueva fase, la de reconocimiento, la de darse cuanta de lo tremendo del problema que se les ha venido y como 
consecuencia, un cúmulo de sentimientos y sensaciones confusas. Los padres se sienten abrumados sintiéndose incapaces de educar a su hijo.

Otro sentimiento común es el de confusión: reciben unas informaciones con una terminología desconocida que les produce temor, porque no llegan a saber hasta que punto alcanza la gravedad del problema.

Posiblemente el sentimiento más generalizado en los padres sea el de culpa (Basseda, 1991). Ellos precisan buscar la causa de la dificultad del hijo. En ocasiones se culpabiliza a los familiares, al poco cuidado de la madre durante el embarazo, a los médicos por la mala asistencia durante el parto, etc.

Para calmar este sentimiento de culpa los padres en contrapartida, protegen en exceso al niño.

La negación como mecanismo de defensa, reduce la ansiedad, pero no impide que los padres sigan buscando otros diagnósticos.

La secuencia siguiente es la aceptación o admisión de la minusvalía del hijo, no sólo a nivel individual sino ante la sociedad que le rodea. Los padres además de aceptar la deficiencia de su hijo, se adaptan e inician actividades constructivas. Entre ellas reordenar su estilo de vida.

Como fase final de este proceso hay que considerar las expectativas de los padres. Estos acuden de un especialista a otro para ver qué esperanzas les proporcionan: nuevas técnicas, implantaciones de prótesis, adelantos farmacológicos y/o quirúrgicos, etc. Por parte del especialista lo mejor es que ayuden a los padres para que aprendan a vivir con la realidad y dejar esas expectativas.

Los profesores que atienden a niños con NEE tienen que afrontar una mayor preparación. Deben poseer, si quieren trabajar con eficacia, amplios conocimientos en el área de trabajo, así como el prestigio y la confianza consiguientes. Deben por tanto estar especialmente adiestrados para colaborar eficazmente con médicos y otros profesionales (Cabrerizo y Pacheco, 1990).

La participación de la familia no sólo es importante en cuanto que colabora en la elaboración del diagnóstico y su puesta al día, sino que también, ella misma es el sujeto del diagnóstico. Las deficiencias se generan allí donde hay un sistema patógeno.

A menudo el problema del niño deficiente no es un problema individual sino el resultado de un mal funcionamiento de todo el sistema. La familia ha de estar por tanto presente, tanto en el diagnóstico como a lo largo de la intervención como cliente de la intervención y/o como agente del desarrollo de los programas preventivos y correctivos.

Las ayudas educativas se van retirando poco a poco, a medida que avanza el niño deficiente en edad. Los padres finalmente, se quedan solos frente al problema. No sólo sus hijos no alcanzan la normalidad, sino que generalmente, la sociedad carece de mecanismos de ayuda y acogida de los sujetos con deficiencias una vez acabada la escolaridad, y pasan, de este modo, a depender exclusivamente de la familia y reaparecen en ella, tanto el pesimismo como los sentimientos de desesperanza. La escuela sería la encargada de amortiguar estos sentimientos dolorosos, preparando a los padres y orientándolos hacia organismos de acogida profesional y social (Casanova, 1990).

\section{Escolarización de niños con necesidades educativas especiales}

La escolarización de un niño/niña con NEE, exige previamente una valoración por parte de los equipos interdisciplinares del sector.

El informe que elabora el equipo determina las NEE del niño. A partir de él comienza la elaboración de la "Adecuación Curricular Individualizada” (A.C.I.), por 
parte del maestro del aula, del maestro de apoyo y otros especialistas, con asesoramiento de los miembros de los equipos.

En las A.C.I. se contempla la participación de los profesionales implicados en el proceso de aprendizaje y desarrollo del alumno, además y especialmente, los padres junto a otros especialistas.

¿Qué tipo de colaboración pide el maestro a los padres? Indudablemente el maestro/maestra no consensúa con los padres que le sustituyan en su función como docente, pero si es claro que los padres pueden crear las condiciones favorables para que el niño esté en disposición de interactuar en su proceso educativo. Una de las primeras condiciones es la de reconocer que el niño con déficit es capaz de realizar actividades en función de su déficit, pero no menos.

Brennan (1990) recoge las siguientes aportaciones de los padres al currículo:

- Proporcionan información sobre las NEE del niño.

- Informan sobre las circunstancias familiares.

- Transmiten información sobre la conducta del niño fuera de la escuela y su actitud hacia la escuela y los profesores.

- $\quad$ Alertan a la escuela sobre cualquier interés o talento que el niño muestra en casa.

- Dan a conocer sus ideas sobre la educación del niño, y su relación con planes posibles y futuros.

- Contribuyen a asegurar la armonía entre el hogar y la escuela.

- Participan activamente en el aprendizaje del niño.

- Organizan visitas familiares que contribuyen al currículo.

- $\quad$ Cooperan con otros padres y profesores en la discusión y evaluación del currículo, los recursos de la escuela, etc.
- Usan su influencia y experiencia para crear lazos entre la escuela y la comunidad.

- Ponen sus conocimientos o habilidades especiales a disposición del currículo escolar.

La evaluación del alumno en el contexto de enseñanza aprendizaje es una tarea compleja que puede facilitarse, mediante la colaboración familiar. De la evaluación del niño se determinará sus NEE, siendo un elemento curricular primordial para la toma de decisiones.

Las informaciones relevantes que ayudarán a conocer mejor al alumno, objetivo este del proceso evaluador, en las que la familia puede aportar datos cruciales, pueden agruparse según Figueroa (1991) en dos grandes bloques:

\section{Información sobre el alumno:}

- $\quad$ Aspectos de su desarrollo.

- Nivel de competencia curricular.

- $\quad$ Estilo de aprendizaje y motivación para aprender.

\section{Información relativa al entorno del alumno:}

- Contexto escolar.

- Contexto sociofamiliar.

Analicemos más detalladamente aquellos aspectos en los que la familia puede aportar información relevante para la toma de decisiones (Vila, 1990):

\section{Información sobre el alumno:}

- Desarrollo general del niño: para los alumnos con NEE, no es suficiente en muchos casos determinar su nivel de competencia curricular para ajustar 
la respuesta educativa. Por lo que es preciso evaluar ciertos aspecto de su desarrollo:

- Aspectos biológicos: datos del tipo médico que ofrezcan información para la determinación de actuaciones concretas.

- Aspectos intelectuales: las capacidades básicas que posee y que serán de utilidad en la planificación de la respuesta educativa y en la orientación de la acción para el desarrollo de dichas capacidades.

- Datos sobre el desarrollo motor: posibilidad de desplazamiento, control postural, capacidad manipulativa, información que ofrecerá pautas para tomar decisiones.

- Aspectos comunicativos-lingüísticos: el nivel fonológico, morfo-sintáctico, léxico y pragmático de su lenguaje oral o el empleo alternativo o complementario de otro sistema comunicativo.

- Información sobre su adaptación o inserción social: relaciones con adultos y otros niños.

- Aspectos emocionales: autoestima, grado de bienestar, grado de confianza en sí mismo y en los demás..., datos relevantes por su repercusión en el aprendizaje.

- $\quad$ Nivel de competencia curricular: lo que implica determinar lo que es capaz de hacer el alumno en relación a las diferentes áreas y objetivos del currículo. En este ámbito los padres pueden colaborar de manera indirecta, pues los equipos interdisciplinares lo encargados de su delimitación.

Pueden ayudar a contrastar el desarrollo conseguido en diferentes situaciones contestar cuestionarios preparados para tal fin, pues ellos pueden observar al alumno en situaciones naturales para completar la observación de los docentes o especialistas.

- $\quad$ Estilo de aprendizaje y motivación para aprender:

- Condiciones físico-ambientales en las que trabaja con mayor comodidad.

- Preferencias relacionales ante las tareas escolares: necesita estar solo para estudiar o acompañado, prefiere el apoyo de adultos o iguales...

- $\quad$ Actividad y contenidos hacia los que se encuentra más interesado.

- Capacidad y nivel de atención.

- $\quad$ Estrategias para la resolución de tareas: reflexibilidad/impulsividad, recursos que utiliza, ritmos de aprendizaje...

- Motivación: metas que persigue, atribuciones frente al éxito o el fracaso.

\section{Información relativa al entorno del alumno:}

- Contexto escolar: en cuanto al entorno escolar los padres pueden ofrecer su opinión sobre la oportunidad de las adaptaciones de acceso físico y/o la comunicación, la propiedad de las 
medidas de atención a la diversidad ofertadas en el centro.

En todo proceso de reflexión resulta fundamental la aportación de otras personas, situación que debe concebirse en un contexto de cooperación y confianza mutua, donde se pretende la mejora del formativo y no la descalificación.

- Contexto sociofamiliar: los familiares son figuras significativas que condicionan el desarrollo del alumno. Así, es necesario recoger información sobre los aspectos más relevantes del medio familiar y social cercano, puesto que aportan elementos útiles para la toma de decisiones curriculares.

Los instrumentos que pueden contribuir a obtener esta información son principalmente, los cuestionarios, herramientas elaboradas por el propio equipo docente o comercializados. El procedimiento clave será la entrevista con los padres y en ocasiones con el propio alumno.

Una vez recogidos lo datos es preciso analizar los condicionantes que favorezcan y los que dificulten el proceso de enseñanza-aprendizaje. Posteriormente, y en colaboración con la familia se ha de intentar ajustar o modificar lo esencial, para así ofrecerle la respuesta más adecuada a sus necesidades.

La información más útil para la toma de decisiones en este campo se puede agrupar según Tena (1991) en torno a tres grandes bloques:

- El alumno: grado de autonomía, hábito de aseo, alimentación..., medio de comunicación que utiliza en casa, interacciones que establece o mantiene, sus aficiones y preferencias, etc.

- La propia familia: número de miembros, rutinas, pautas y hábitos familiares, expectativas frente a sus hijos, conocimiento de las posibilidades reales que tiene el alumno, grado de colaboración que se espera, expectativas en relación al centro..., entre otros aspectos.

- El medio social: Características y recursos existentes en la vivienda, servicios de la comunidad, prestaciones que reciben o pueden recibir de autonomía e inserción social que ofrecen..., con vistas a planificar experiencias escolares que potencien su integración social.

Este análisis ofrecerá pautas para elaborar y poner en marcha programas de intervención educativa lo más ajustados posibles a las necesidades específicas del alumno.

En un principio en general, la escolarización de niños con NEE en los centros ordinarios (y dentro de ellos en aulas ordinarias), aunque ello no es siempre posible, bien por las características diferenciales del alumno/alumna, bien por los medios con los que se cuenta en el centro educativo.

Es obvio que la participación de los padres en la elección del centro es vital, aunque ese derecho viene limitado por la legislación, en función directa de la valoración psicopedagógica que los equipos de orientación lleven a cabo.

\section{Colaboración entre Institución educativa y familia}

Es un derecho de los padres el ser informados e incluso formados acerca de la mejor educación que se da o deben dar a sus hijos en un marco interactivo.

Siguiendo a Corrandini (1997) podemos destacar las ventajas de esta colaboración:

La escuela gana al conocer las expectativas de los padres y sus reacciones. Es una obligación de la escuela el conocer esas expectativas que pueden estar o no de acuerdo con las posibilidades del niño. 
Los padres sacan provecho al conocer la problemática de sus hijos y cómo éste se desarrolla. Del conocimiento de estos factores surge la relación permanente entre padres y maestros y conduce a una ayuda recíproca, y a una mejora de los métodos, pues la escuela se aproxima a la vida real y los padres se aproximan a la vida escolar, ya que tanto padres como profesionales buscan los mismos objetivos y de ahí puede surgir el distribuirse responsabilidades.

Esto ocurriría en cualquier relación padres/profesores, cuanto más surgirán cuando el alumno está necesitado de una ayuda más específica debida a sus NEE.

Si hay una buena interrelación de la familia con el profesor, ésta le manifiesta sus dudas, angustias, y el profesor puede prestarle apoyo para superar esa situación emocional, aunque sólo sea escuchándoles.

Es necesario orientar a los padre, ya que a veces las familias de niños con discapacidad tienden a distanciarse entre ellas y con los demás miembros de la comunidad; por lo tanto, se hace necesario romper barreras acercándose a ellas de manera respetuosa, teniendo en cuenta que mejorar a la familia que viva la experiencia de tener a una persona con NEE, es mejorar y ganar terreno en la educación del niño con NEE, porque no hay que olvidar que uno de los objetivos en el trabajo de estos niños, es su integración social y ésta empieza en la familia y en su entorno.

Al mismo tiempo hay que trabajar con la familia para que en lo posible le den autonomía al niño con discapacidad.

La escuela y la familia son para el niño/niña el medio principal en el que encuentra alimento cotidiano su conducta. La génesis de las conductas y las repercusiones mayores se producen en el ámbito familiar y escolar. Estas dos esferas de la vida dentro de las que el niño interactúa, obliga a una coparticipación de responsabilidades. La intervención psicopedagógica, si ha de ser efectiva, reclama la inclusión y tratamiento de todo el sistema familiar.
La escuela por medio de sus profesores está obligada a interactuar con el medio familiar en el que residen la mayor parte de los recursos personales y motivacionales de la conducta. Esta participación en la intervención se hace tanto más necesaria cuanto más joven es el niño. Es en la casa donde se encuentran la mayor parte de las situaciones naturales y estimulantes que permiten el desarrollo de las acciones preventivas primarias y secundarias. Esta acción educativa del sistema familiar exige un tratamiento desde múltiples planos (Ríos, 1986).

- Un plano educativo que requiere sistematización de lo formativo, cuerpo doctrinal que transmitir, ideas eje sobre las que incidir de forma más concreta, instrumentos de autoformación asequibles para los más interesados y posibilidad de contrastar experiencias e ideas que contribuyan a un permanente y progresivo enriquecimiento de los usuarios.

- Un plano orientador, en cuanto que a partir de las situaciones reales y concretas de cada S.F. la interacción entre la familia y los agentes formadores engendre una potenciación de capacidades, un descubrimiento de nuevas partes, y la estructuración de códigos nuevos para cada familia.

Un plano terapéutico que lleve consigo nuevos modos de comportamiento intrafamiliar, reestructuración de las relaciones, comunicaciones, intercambio de mensajes, redefinición de metas y construcción de un nuevo estilo educativo que integre cuantos factores personales, ambientales o situacionales puedan contribuir a un mayor nivel de higiene mental como totalidad.

La escuela no puede continuar creyendo que sólo ella tiene el poder educador. Hay que devolver a la familia la capacidad 
terapéutica implicando a todos los miembros del sistema.

El enfoque educador habrá de adoptar enfoques sistémicos en vez de explicar los problemas del sujeto especial como un suceso aislado. Para ello la primera condición exigida es la formación en el ámbito de la orientación familiar del propio profesional de la intervención, cualquiera que sea su nivel.

Uno de los mayores problemas, en la no siempre feliz interacción entre la institución educativa y la familia del niño/niña con Necesidades Educativas Especiales, suele ser la existencia de una serie de prejuicios mutuos; por lo que a nosotros respecta, no es raro que manifestemos hacia los padres lo que Vidal y Díaz (1990) señalaban:

\section{a) Los padres como clientes vulnerables}

A menudo solemos pensar en los padres de nuestro alumno con NEE como personas con escasa capacidad de respuesta ante sus problemas, y como consecuencia, más como un objeto de nuestra composición que como adultos que pueden y deben enfrentar una situación más o menos problemática.

\section{b) Los padres como pacientes}

Tampoco es raro que desde una ciencia similar, pensemos en los padres como individuos que necesitan, ellos mismos, algún tipo de atención psicológica. Con independencia de que ello pueda ser más o menos cierto en algunos momentos (la necesidad de esta ayuda se entiende), los padres no son nuestros pacientes, sino personas en plenitud de sus derechos y sus facultades, que debemos tratar como tales.

\section{c) Los padres como culpables}

Los prejuicios anteriores alternan con las creencias de que muchas veces los padres son los culpables de la situación del alumno, a consecuencia de su inadecuada disposición, falta de interés u otros motivos, se les trata de modo que se profundiza en posibles sentimientos de culpa que a veces acompañan al nacimiento de un hijo con discapacidad. No parece que sea el mejor modo de contribuir a la educación y el desarrollo de nuestros alumnos.

\section{d) Hay que mantenerse a distancia}

Nuestro trabajo implica un contacto emocional bastante intenso con los alumnos y sus familias, de modo que a veces tendemos a protegernos, tratando de mantener una relación más o menos distante. El único problema es que es bastante difícil la cooperación entre personas cuando uno de los polos percibe que el otro no se siente comprometido con el asunto porque "no es su problema".

\section{e) Los padres como poco capacitados}

¡Nosotros somos los técnicos! No es raro escuchar esta expresión y otros comentarios acerca de la insolencia de los padres que se atreven a opinar en lo que no son expertos. Sin embargo pensamos en nuestros propios hijos y en qué medida somos conscientes de sus necesidades, tenemos expectativas sobre ellos, conocemos cómo reaccionan ante diversos estímulos.

\section{f) Los padres como adversarios}

El prejuicio anterior suele ir acompañado de éste $y$, con independencia de que éste así ocurra, lo cierto es que un enfrentamiento requiere siempre de dos contendientes al menos: la perspectiva sistemática, se ha dicho ya, supone que todos los puntos de vista son legítimos y no deben ser enfrentados, sino confrontados y negociados.

\section{g) Tendencia a catalogar a los padres}

El mayor error es la tendencia a etiquetar a los padres en categorías simples 
y, siempre, inapropiadas (tanto como las de ellos hacia nosotros), ya que mediatizan nuestras interacciones e interfieren en nuestra cooperación.

Frente a estos prejuicios los mismos autores a los que venimos citando proponen el sano ejercicio de tratar de ponerse en la piel de las familias cuyo hijo presenta NEE vinculadas con algún tipo de handicaps más o manos importantes; con independencia de las diferencias enormes, de un caso a otro. Es posible comprender mejor su vivencia y su posición, si tenemos en cuenta que se enfrentan a un conjunto de retos o desafíos nada sencillos de afrontar, como pueden ser:

La necesidad de implicarse activamente en la enseñanza sistemática de habilidades y destrezas de su hijo, en la medida en que éste no aprenda de manera tan espontánea por inmersión en las actividades cotidianas en el hogar y en la comunidad.

- A menudo deben extremar el papel de "asesor" inherente a todo padre y madre en el sentido de que, con frecuencia, su hijo se ve expuesto a vivencias y sentimientos de inferioridad e incluso rechazo, especialmente en la adolescencia; los padres se enfrentados a situaciones en las que no resulta en absoluto fácil salir airoso y ayudar a la vez al crecimiento emocional del joven.

- $\quad$ En cuanto que las conductas inadaptadas, tienden a ser más frecuentes cuanto menor es la capacidad comunicativa y de interacción del individuo. Las familias del niño con discapacidad deben también aprender a hacer frente a ellas, y a las consecuencias que implican en la vida cotidiana de la familia.

Al mismo tiempo deben educar y atender emocionalmente a sus demás hijos que no es raro que se vean envueltos -cuando son más pequeños-en sentimientos contradictorios, pues comprenden que su hermano o hermana precisa mayor atención (incluso de su propia parte), pero no pueden dejar de experimentar los sentimientos, si no de abandono, sí de mayor descuido por parte de los padres. Parte importante de esa educación de los demás hermanos es ayudar a comprender cómo deben actuar para contribuir al desarrollo apropiado de aquel, tarea que se extiende también a los tíos, abuelos y demás personas significativas en la vida del niño con discapacidad.

- Tampoco es raro que las tensiones que a menudo suponen todo lo anterior, afecten la relación de la pareja: un factor más al que enfrentarse y afrontar.

- $\quad$ Por si todo lo anterior fuera poco, deben comprometerse aún en mejor grado que las demás familias en la educación escolar de sus hijos, así como en agrupaciones ciudadanas para la defensa de sus derechos.

\subsection{Relación familia/ maestro}

El maestro/maestra para los padres y madres es un especialista y a menudo un rival y a veces trata de pasarle la responsabilidad. Con frecuencia los educadores, sin querer asumen el papel de la "buena madre" o "buen padre" que sabe hacerse obedecer por el niño y sabe "sacar algo bueno de él", lo que no hace más que aumentar la tendencia de la mayor parte de los padres naturales de sentirse culpables o impotentes ante las dificultades de su hijo.

Se trata de conseguir lo contrario, reforzar su propia imagen como padres, valorándola como valiosa e insustituible, diferente a la de cualquier adulto. El maestro no es sustituto de la familia. 
El profesor ve a veces a los padres como una carga, porque interfieren en lo que ellos enseñan. Por ello hay que aclarar esas ambigüedades y hacer peticiones $\mathrm{y}$ demandas específicas de padres a maestros y de maestros a padres.

Siguiendo a Álvarez (1987), podemos indicar que los padres y madres necesitan:

a) Información específica sobre su hijo, cómo le afecta el problema y cómo avanza escolarmente.

b) Explicarles claramente cómo pueden colaborar. Para ello sería bueno llevar un cuaderno de trabajo en el que el profesor tutor o de apoyo expresará qué experiencias concretas pueden trabajar con su hijo, coordinadas con lo que se hace en clase para afianzar los conceptos que se trabajan en la misma.

Es aconsejable poner a los padres de niños con dificultades en contacto, pues así se ayudan.

c) También sería útil que los padres vean cómo sus hijos participan y trabajan en el colegio, pues a veces tienden a valorarles ellos mismos, y además esto les sirve de modelo para trabajar ellos.

Algunas experiencias positivas aconsejan que los padres puedan entrar en las clases de sus hijos o en las sesiones de logopedia, por ejemplo, para que observen cómo trabajan los niños; así pueden ser más objetivos en la valoración y aprender modelos de trabajo.

También favorecerá la interacción padres/maestro, el fijar el ámbito físico donde se realizará el prólogo y el desarrollo de la intercomunicación, es decir, de los primeros y sucesivos encuentros; además de establecer el tiempo en el que se van a hacer efectivos.
A medida que el sistema escolar avanza sobre terrenos, antes pertenecientes al ámbito exclusivo de la familia, crecen en importancia las estrategias que se ponen en juego para establecer lazos de unión entre los programas educativos escolares y los de la familia.

Esta relación es particularmente importante en las primeras etapas en las que debe darse una continuidad sin fisuras entre la atención educativa escolar y la familiar.

Hoy que la educación infantil comienza a los 3 años, y teniendo en cuenta que los niños deficientes están generalmente ya integrados en las aulas ordinarias, es más necesario que nunca desarrollar estrategias concretas que conviertan esta relación en una relación efectiva y no solo en la relación padres y maestro.

Programas que antes eran de carácter preventivo, fundamentalmente familiar, pasarán a formar parte principal de la educación ordinaria escolar.

La institución escolar, sin embargo, opone aún ciertas resistencias a ofrecer a los niños con deficiencias una atención temprana suficiente. Ello es un síntoma que revela un cierto recelo del sistema educativo ordinario a recibir a estos alumnos, que, de este modo pierden los mejores momentos para su educación. Son estos momentos por tanto los más difíciles y los más necesitados de orientación y ayuda. A menudo cuando faltan medios materiales son estos alumnos los primeros en sufrir estas carencias, la dificultad para conducir programas educativos es más evidente en estos momentos primeros. La escuela no debiera dejar a los padres sin ayuda cuando más la necesitan.

Si a ello añadimos la dificultad de que muchas anomalías están ligadas al ambiente deprivado educativamente en el que vive el niño, se comprende mejor la necesidad de estrechar lazos entre el educador y los padres con menos capacidades.

Las asociaciones de padres y las recomendaciones internacionales apuntan hacia una pronta acogida de los niños deficientes a 
los que antes, con diversas excusas (control de esfínteres, problemas motores), se impedía incluso el acceso físico.

Así pues, los programas de escolarización deben ser completados, para que esta educación tenga sentido, con una asistencia a los padres para que estos puedan ayudar eficazmente a sus hijos. Ningún aspecto de la educación deficiente es más importante que éste (Warnock, 1990). La tecnificación del profesorado en esta asistencia debiera pasar a formar parte sustancial de su formación, más en las condiciones actuales en las que la educación especial no se define ya por el lugar donde debe impartirse, sino por las necesidades que ésta debe satisfacer.

Las relaciones escuela-familia son reducidas en general, ya que la participación de los padres se suele limitar a aportar alguna información poco relevante porque, a menudo, el sistema escolar, incluidos los profesionales de la intervención psicopedagógica, tienen escasa costumbre de trabajar con las familias. Esta falta de colaboración se debería según Ríos (1972, 1979, 1986), a las siguientes causas:

- A menudo hay una falsa perspectiva de lo familiar: en ocasiones se piensa que la familia puede aportar muy poco y que ni siquiera sería capaz de enfrentarse con los aspectos disfuncionales que le afectan a ella misma. No se da a los padres la posibilidad de manifestar sus propias necesidades y exponer sus conflictos en un contexto de comprensión y seguridad de que recibirán la ayuda precisa. El hecho de dejarla de lado, supone una sobrevaloración de las técnicas psicopedagógicas y por tanto del poder del educador o del especialista, lo que lleva a la familia a convencerse de su incapacidad para resolver sus propios conflictos.

La presencia de tensiones emotivas generadas por la distancia y desconocimiento mutuo: se produce general- mente el sometimiento del sistema familiar al sistema escolar por lo que los contactos son meramente superficiales.

Analicemos a continuación algunos de los cauces de participación y colaboración entre padres y profesores que nos da Buscaglia (1990). Podemos diferenciar formas de participación individual y colectiva:

\section{A) Cauces de participación individual}

Los objetivos de estas formas de colaboración familiar en la vida del centro son:

- Posibilitar el conocimiento del alumno y de su familia.

- Intercambiar información sobre el alumno.

- Asesorar y orientar a los padres y madres de los alumnos/alumnas.

- Facilitar la incorporación y posterior adaptación del niño al medio escolar.

\section{a) La entrevista}

Esta estrategia permite el acercamiento directo entre la familia y el centro. Se ha de destacar por su importancia la entrevista inicial, en este primer encuentro, padres y educador centrarán las bases de la futura relación, iniciándose de mutuo conocimiento que posibilitará el establecimiento de un clima de confianza. En ella el educador obtiene directamente referencias sobre el niño y su familia.

Aunque anteriormente el profesor ha podido consultar informes de otros profesionales del ámbito clínico-sanitario y educativo. Los datos recogidos, aunque de carácter general, servirán para orientar las futuras relaciones bilaterales y encaminar 
el proceso educativo del alumno, en el que los padres se verán implicados.

A su vez, en la entrevista inicial, los padres obtendrán información sobre el centro, sus servicios, objetivos, recursos personales y materiales, espacios, y en definitiva medios didácticos y organizativos de los que dispone el colegio.

Con entrevistas posteriores, cuya temporalización ha de consensuarse, se contribuirá a:

- $\quad$ Detectar dificultades de aprendizaje y/o adaptación.

- Plantear soluciones a los problemas que puedan surgir.

\section{- Intercambiar información.}

- Adaptar objetivos y estrategias de intervención comunes.

- Orientar y asesorar a las familias sobre temas puntuales.

Aseguran, mediante el seguimiento, la retroalimentación del proceso, facilitándose considerablemente la labor del educador (Coll, 1990). Y en los padres se establece una relación de confianza y seguridad.

Si bien, la entrevista es considerada como una de las técnicas de relación y participación más eficaz, ha de ser complementada con otras vías de participación individual.

\section{b) Encuentros informales}

Estos encuentros informales se pueden planificar a las entradas y a las salidas, ya que los niños llegan y se van acompañados. Lo más conveniente es preverlos dentro del horario del centro, para evitar alteraciones en la dinámica del aula.

Esta fórmula posee la ventaja de aumentar la naturalidad y facilitar la continuidad de la relación profesores-padres.
Los encuentros informales incrementan su eficacia si están incluidos dentro del Proyecto de Centro.

\section{c) Voluntariado}

Constituye la incorporación de los padres y otros familiares a las tareas diarias de la escuela. Este tipo de colaboración supone un apoyo directo para los profesores y cuidadores, y requiere una planificación conjunta del trabajo que es posible realizar.

Las posibilidades son diversas:

Planificación y organización de salidas extraescolares.

Preparación de fiestas y actos culturales y deportivos.

Diseño y ejecución de situaciones de aprendizaje.

Elaboración del material.

Acondicionamiento de espacios.

Participación en talleres, entre otras fórmulas de colaboración.

Las ventajas son importantes:

Aumento de los recursos humanos, con la consiguiente reducción de la ratio profesor/alumno.

- $\quad$ Autosatisfacción de los padres y madres al sentirse útiles y necesarios.

Satisfacción de los niños al contemplar la participación de sus padres y los resultados de la misma.

d) Otras formas de colaboración individual

Circulares: facilitan el intercambio unidireccional de información, son 
válidas para transmitir contenidos de carácter divulgativo que no necesitan respuesta.

- Llamadas telefónicas: son útiles para ofrecer o recibir información más personalizada principalmente cuando los niños, por su edad o características individuales, no pueden comunicárselo a sus padres personalmente. Son convenientes para transmitir información muy puntual y de carácter urgente.

- Informes escritos: han de presentar la información con claridad, concreción y brevedad. Han de redactarse en sentido constructivo, aunque realista.

\section{B) Cauces de participación colectiva}

La participación colectiva de los padres y madres de alumnos en el centro, pretende entre otros los siguientes objetivos:

- Posibilitar el intercambio de experiencias entre las distintas familias.

- Facilitar el conocimiento del grupo al que pertenece el alumno.

- Economizar esfuerzo en los temas de interés común.

- Promover lugares para la reflexión y el encuentro.

- Participación en la gestión del centro educativo.

El papel de las Asociaciones de Madres y Padres de alumnos/alumnas (AMPAs) será decisivo. Siempre y cuando se utilice su estructura para informar, consultar y recoger las inquietudes de las familias. La participación mediante la AMPA no se limitará a los aspectos administrativos, sino que serán vehículos de animación sociocultural y de dinamización y actividad del centro.
El centro se enriquece y completa su proyecto curricular, gracias a las actividades en que se desarrollan en las asociaciones de padres y madres de alumnos/as (CEFAES, 1988). A estos grupos pertenecen todos los padres y madres que así lo desean, entre los que se elige democráticamente a quienes van a representarles en el Consejo Escolar.

\section{a) Representación en consejos escolares}

Una de las premisas que condicionan la eficiente participación de los padres, es la adecuación de la dinámica interna de los Consejos Escolares a los intereses y necesidades de los sectores representados. El contenido del Consejo Escolar debe ser así equilibrado y contener los problemas que preocupan a todos los miembros de la comunidad educativa. Por consiguiente la metodología será participativa, ha de ofrecer a todos sus componentes la posibilidad de expresar sus opiniones.

Es importante saber que en las reuniones del Consejo Escolar deberán celebrarse en el día y hora que posibilite la asistencia de todos sus miembros y, en todo caso, en sesión vespertina. En las reuniones ordinarias, el Secretario o Secretaría del órgano colegiado, por orden del Presidente o Presidenta, convocará con el correspondiente orden del día a los miembros del Consejo Escolar, con una antelación mínima de una semana y pondrá a su disposición la correspondiente información sobre los temas incluidos en el mismo.

La eficacia del Consejo Escolar dependerá de la evitación de factores como los siguientes (Ajuriaguerra, 1985):

- Parcialidad.

- Corporativismo.

- $\quad$ Creación de bloques antagónicos.

- No valoración de las reuniones. 
- Inoperatividad.

- Inexistencia de comisiones de trabajo, etc.

Por el contrario, funcionará si se favorece:

- $\quad$ Fluidez de información veraz.

- Negociación de las posturas.

- $\quad$ Análisis de todas las propuestas.

- Evaluación de los resultados y del propio proceso.

- Concreción de acuerdos, etc.

\section{b) Reuniones de padres}

Las reuniones de padres podrán ser convocadas por el delegado de padres del curso o por el docente en sí. Los principales objetivos que persiguen son:

- $\quad$ Transmitir los objetivos, actividades y evaluación de la concreción, que sobre el Proyecto Curricular de Centro han planificado los profesores y profesoras para el grupo de alumnos/alumnas.

- Explicar las dificultades que es posible que se encuentren sus hijos en el proceso de enseñanza-aprendizaje previsto para el curso.

- Comunicar las estrategias que se pueden utilizar para satisfacer las necesidades educativas de los alumnos.

- Difundir los detalles organizativos, horarios, materiales que deben adquirirse, consultas..., entre otros aspectos.

En estas reuniones, sin mermar la naturalidad y comunicación espontánea, se ha de cuidar su planificación previa, para evitar la accidentalidad.
El éxito de la reunión estará condicionado por factores como los que a continuación se exponen:

- Disposición de los asistentes.

- $\quad$ Presentación de los mismos.

- Valoración de la asistencia de los presentes.

- Clarificación del objetivo de la reunión.

- Facilitación de vías de participación de todos los asistentes.

\section{c) Escuelas de padres y madres}

La Escuela de Padres y madres es una organización cuyo objetivo inicial es la búsqueda de información para un mejor entendimiento entre padres y profesionales de la educación de cara a evitar posibles conflictos y malentendidos (Mittler, 1991). Pero aunque padres y profesores deban colaborar en una misma tarea educativa, debemos tener en cuenta la delimitación de objetivos y competencias de las partes implicadas.

Los objetivos de esta forma de participación basándonos en Hegarty y otros (1988):

Mejorar las relaciones padres/madres-hijos.

- Facilitar un clima de confianza, respeto y estima entre los miembros de la familia.

Promover actitudes positivas hacia el hijo.

Desarrollar habilidades específicas.

Potenciar el intercambio de experiencias y respuestas a los problemas o situaciones conflictivas planteadas. 
- Dinamizar la participación de los padres y madres en la escuela.

Un factor que contribuirá al éxito del funcionamiento de las Escuelas de Padres será que sean dirigidas, coordinadas y gestionadas por los propios padres. Aunque naturalmente contando con el apoyo y asesoramiento de los profesores y técnicos del centro.

\section{d) Otras formas de colaboración} colectiva

- Comisiones de Trabajo: se relacionan con los órganos directivos estrechamente, siendo su contacto con los profesores menos directo aunque eficaz, puesto que ayudan a resolver problemas concretos y a mejorar la infraestructura del centro. Estas comisiones se constituirán en función de las necesidades del centro. Igualmente contribuyen al establecimiento de las relaciones con organismos públicos e instituciones sociales del entorno (Hinojo y Aznar, 1997).

- $\quad$ Reuniones de Tipo Festivo: en la que participan todos los miembros de la comunidad educativa. Sirven para establecer contactos más estrechos, cálidos y cordiales entre los diversos sectores de la escuela. Se realiza con ocasión de fiestas de carácter variado.

- Otros cauces de participación colectiva lo constituyen las revistas del centro, los boletines informativos, las charlas y conferencias, etc.

\section{Consideraciones finales}

Según se ha desarrollado en este artículo, el trabajo coordinado entre los maestros y los padres de familia de las personas con NEE, puede favorecer no solo la atención adecuada de los estudiantes, sino la comprensión por parte de ambos de las necesidades, características e intereses que tienen las personas cuando se involucran en un proceso de aprendizaje.

También, un trabajo compartido o colaborativo entre los padres de familia y los docentes, permitiría el desarrollo de procesos de sensibilización y concientización con respecto a las diferencias individuales de todas las personas, favoreciendo de esta manera, la integración de los estudiantes con NEE a las sociedad y las instituciones educativas.

Los procesos de escolarización de los estudiantes con NEE requieren por lo tanto, el conocimiento de las características y las potencialidades de estos estudiantes, así como sus necesidades e intereses, de tal manera que el aporte de los padres a este conocimiento es invaluable, ya que son quienes mejor conocen a sus hijos.

Teniendo en cuenta lo anterior es que se propone en este artículo la importancia de realizar un diagnóstico integral de la persona, pero desde un enfoque sistémico, o sea que se tomen en cuenta todos aquellos aspectos que afectan a la persona, tanto en la comunidad, como en la familia, la institución, el grupo y la persona con NEE, ya que se parte de la idea de que como anota Soto (2002) la persona con NEE, presenta sus necesidades en tanto, tiene que realizar un proceso de interacción con personas, grupos o docentes que le proponen un proceso de aprendizaje, en el cual muchas veces no se toman en cuentas sus particularidades, situación que podría generar una dificultad de acceso a los procesos de aprendizaje del estudiante.

Es por lo anterior, que es imperante la necesidad de realizar procesos de diagnóstico de manera integral, en donde se tomen en cuenta todos los sistemas y subsistemas en los que comparte el estudiante, los cuales se ven afectados por él, y viceversa.

Por lo tanto, el docente y el padre de familia deben tener una posición clara de 
cúal es el papel que cada uno de ellos debe cumplir en el proceso de atención a la diversidad en las instituciones educativas, esto se podría ver potencializado en el momento en que se establezca una colaboración entre docentes y padres de familia, teniendo en cuenta que los padres pueden incorporarse al trabajo de aula y al desarrollo de proyectos institucionales, lo cual favorecería la integración tanto de la familia como del estudiantes con NEE a las instituciones educativas. En este sentido, uno de los aspectos importantes a considerar es la negociación que se puede realizar entre los docentes y los padres de familia, para de esta manera, dejar muy claro cuáles son las delimitaciones que tiene cada uno en el proceso de apoyo a los estudiantes y a sus hijos.

Para finalizar, es importante recordar que los padres de familia han sido y serán siempre un elemento importante en el desarrollo de los estudiantes, y que si se involucran en los procesos educativos de sus hijos, se enriquecerá la actuación tanto del docente como de la institución en la respuesta a la atención de la diversidad en los centros educativos. Así que es una decisión que debe fundamentarse en el respeto mutuo y el apoyo para el éxito de los procesos educativos que lleven a cabo para construir una educación para todos.

\section{Referencias bibliográficas}

Ajuriaguerra, J. Manual de psiquiatría infantil. Barcelona: Toray-Masson. 1985.

Alvarez, A. "La escuela en la sociedad de hoy: el país de nunca jamás”. En $R e$ vista Cuadernos de Pedagogía, № 147. 1987.

Basseda, E. y otros. Intervención educativa y diagnóstico psicopedagógico. Barcelona: Ed. Paidós. 1991.
Brennan, W. K. El currículo para niños con necesidades especiales. $2^{\mathrm{a}}$ ed. Madrid: Siglo XXI, 1990.

Buscaglia, L. Los discapacitados y sus padres. Buenos Aires: Emecé. 1990.

Cabrerizo, P. y Pacheco, A. Tu hijo diferente. Orientaciones en la familia. Madrid: Palabra. 1990.

Casanova, M.A. Educación Especial: hacia la integración. Madrid: Escuela Española. 1990.

C.E.F.A.E.S. Jornadas nacionales para padres de deficientes mentales: esperanzas objetivas. Pamplona. 1988.

Coll, C. Aprendizaje escolar y construcción del conocimiento. Barcelona: Paidós. 1990.

Corrandini, L. "Comunidad, democracia, pluralismo y participación: el caso de Italia". Revista de Educación, № 252. Servicio de publicaciones MEC. 1997.

Díaz Aguado, M.J. La educación familiar. En J. Beltrán Llera (comp.) Psicología educacional. Madrid: UNED. 1984.

Figueroa Iñiguez, M.J. Nuevas formas de actuación en educación especial. Madrid: Escuela Española. 1991.

Hegarty, S. y otros. Aprender juntos. La integración escolar. Madrid: Ed. Morata. 1988.

Hinojo, F.J. y Aznar Díaz, I. "Los Padres en los Centros Educativos". En M. Lorenzo; F. Salvador y J. A. Ortega (Coords). Organización y Dirección de Instituciones Educativas. Perspectivas actuales. Granada: Grupo Editorial Universitario. 1997. 
Hinojo, F. J. y Aznar Díaz, I. "La participación de los padres en las Instituciones Educativas". En Revista Padres y Maestros, (España) 267, pp. 20-25. 2002.

Mittler, P. "La participación de la familia y la comunidad en el desarrollo del currículo". En Perspectivas de Rehabilitación Internacional. Madrid: Servicio de publicaciones INSS. 1991.

Pelechano, V. "Terapia familiar comunitaria”. En Monográficas del Departamento de Psicología evolutiva y diferencial. Valencia: Alfaplus. 1980.

Ríos González, J.A. Familia y centro educativo. Madrid: Paraninfo. 1972.

Ríos González, J.A. "La terapia relacional en el subsistema conyugal”. En $E d u$ cadores, № 104. 1979.

Ríos González, J.A. "Orientación familiar". En Santiago Molina y otros.
Enciclopedia temática de la educación especial. Madrid: CEPE. 1986.

Soto, R. Didáctica del lenguaje y de los Estudios Sociales para personas con necesidades educativas especiales. San José, Costa Rica: Editorial de la Universidad Estatal a Distancia (EUNED). 2002.

Tena Martínez, E. El entorno sociofamiliar del niño deficiente. En $\mathrm{Ne}$ cesidades Educativas Especiales. Manual teórico práctico. Málaga: Aljibe. 1991.

Vidal Lucena, M., Díaz Curiel, J. Atención temprana. Madrid: CEPE. 1990.

Vila, A. ¿Quién es diferente?. Convivencia diaria con un niño-a deficiente. Madrid: Narcea. 1990.

Warnock, H. "Informe sobre necesidades educativas especiales". En Revista Siglo Cero, № 130, julio-agosto. 1990. 